\title{
Comparative study between El-Ganzouri airway risk index alone and in combination with upper airway ultrasound in preoperative airway assessment
}

\author{
Mohamed Amr Abo Sabaa, Gamal Farouk Amer, Abd Elwehab Abd Alsatar Saleh, Mohamed \\ Abd ElrahmanAbd Elkader Elbakery
}

Department of Anesthesiology and Intensive Care, Faculty of Medicine, Al-Azhar University

\begin{abstract}
Background: The upper airway is an extraordinarily complex anatomical region. Anticipating and preparing for difficulty in airway management is crucial to avoid airway catastrophes.

Objective: Aim of our study was to evaluate the combination of El-Ganzouri airway risk index (EGRI) with the ultrasound of the airway (measuring the hyomental distance and distance from epiglottis to midpoint between the two vocal cords) as a fast, easy and noninvasive method in the examination of the airway and prediction of difficult airway.

Patients and methods: Preoperative airway assessment will be carried out for 60 adult patients randomly allocated in Al-Azhar University Hospitals.

Results: The hyomental distance at cutoff point of $5.6 \mathrm{~cm}$, (below which the intubation difficulty is high), sensitivity and specificity of Cormack-Lehane grade with hyomental distance in study group was $75.0 \%$ and $94.4 \%$ respectively with accuracy of $85 \%$. For the epiglottis to vocal cord distance the cut off value of $\leq 1.2$ (below which the intubation difficulty is high) the sensitivity of epiglottis to vocal cord distance was $75.0 \%$ and the specificity was $100 \%$ with accuracy of $92 \%$.

Conclusion: We concluded that combination of EGRI with epiglottis to vocal cord distance and hyomental distance is a very sensitive and specific tool for prediction of difficult airway and may be superior to standard methods of assessment.
\end{abstract}

Keywords: El-Ganzouri airway risk combination, Upper airway ultrasound, Preoperative airway assessment

\section{Introduction}

Management of the airway remains a major contributor to death and brain damage in anesthesia, emergency medicine, and intensive care settings ${ }^{(\mathbf{1})}$. Examination of the airway is an essential component of the anesthesiologist's pre-operatively. Several bedside physical airway assessment tests are available including an assessment of mouth opening and dentition, Mallampati classification, thyromental distance measurement and evaluation of neck mobility. These methods are quickly and easily performed at the bedside, but unfortunately, their sensitivity and specificity for accurate prediction of difficulty with airway management is not very robust ${ }^{(2)}$. They may also be difficult to apply in emergency and critical care settings, where patients are frequently confused, uncooperative and unable to follow directions. Despite the availability of multiple airway assessment methods, unexpected difficult intubations occur with a frequency of $1-8 \%{ }^{(3)}$. Other authors defined difficult laryngoscopy as a separate identity that occurs when "it is not possible to visualize any portion of the vocal cords with conventional laryngoscopy." This typically corresponds to a Cormack and Lehane Grade 3 or 4 laryngoscopic views ${ }^{(4)}$.

The rate of complications is affected by definitions used and by the clinical setting. The incidence of failed intubation is $\approx 1$ in 1-2000 in the elective setting, $\approx 1$ in 300 during rapid sequence induction (RSI) in the obstetric setting, and $\approx 1$ in $50-100$ in the emergency department (ED), intensive care unit (ICU), and pre-hospital setting. The rate of CICV requiring ESA may rise to 1 in 200 in the $\mathrm{ED}^{(5)}$.

Various clinical criteria are used to evaluate patient's airway prior to induction of anesthesia like Mallampati classification, Wilson scoring system, Samsoon and Young scoring system 
using anatomical structures like thyromental distance, mouth-opening size, neck extension, jaw protrusion and the upper-lip bite test. But of all these methods, Cormack-Lehane classification has been shown to be more reliable one ${ }^{(\boldsymbol{)}}$.

The ultrasound (US) has been in clinical use since the early 1900s, and its use in airway management has been published since then. Ultrasonography (US) has many potential advantages, it is safe, quick, repeatable, portable, widely available, relatively inexpensive, pain-free, and gives real-time dynamic images ${ }^{(7)}$.

Ultrasonography (USG) of the upper airway is capable of providing detailed anatomic information and has numerous potential clinical applications. It can be used to identify airway pathology and may assist other methods in prediction of difficulty with airway management ${ }^{(2)}$.

\section{Aim of the Work}

\section{1) Primary objective:}

This study aimed to compare the preoperative assessment of the airway using ElGanzouri airway risk index alone and in combination with upper airway ultrasound in visualizing the larynx using Cormack and Lehane grading system in adult patients scheduled for elective surgery.

\section{2) Secondary objectives:}

- Ultrasound assessment of the following:

- Visualize the hyoid bone.

$\circ$ Measure the hyomental distance in neck extended position.

- Measure the distance from the epiglottis to the midpoint of the distance between the vocal cords.

- Assessment of the ease of gas exchange during positive pressure ventilation via face mask after induction of anesthesia and muscle relaxation.

Patients and Methods
Design of the study: Prospective and randomized study.

Setting: In Al-Azhar University

Hospitals, in the elective surgical theaters.

Patients: After obtaining approval from the Research Ethics Committee and written informed consents from all patients undergoing any elective operation in Al-Azhar University Hospitals. Patients were meeting the inclusion criteria then preoperative airway assessment was carried out for 60 adult patients that were randomly allocated into two groups:

$>$ Group I (control group, $\mathrm{n}=30$ ): patients were assessed preoperatively by ElGanzouri airway risk index "EGRI" to detect the possibility of difficult intubation (score $<4$ is unlikely to be difficult and score $\geq 4$ is more likely to be difficult).

$>$ Group II (study group, $\mathrm{n}=30$ ): patients were assessed by EGRI and by upper airway ultrasound.

Inclusion criteria: Patients with ASA physical status I or II were scheduled for any elective surgery requiring endotracheal intubation for general anesthesia.

\section{Exclusion criteria:}

Age $<18$ years old.

$>$ Patients with obvious abnormalities of the airway who should be scheduled for awake intubation (e.g. facial fractures, tumors, etc.).

$>$ Cervical spine fractures.

Tracheostomy tube.

Unable to give consent.

Methods: During the preoperative visit, the techniques have been explained to the patients.

1) Pre-operative assessment:
A) Medical history.
B) Physical Examination.
C) Anesthetic assessment.
D) Laboratory investigations.

\section{2) Demographic data:}

The patient age, sex and ASA status have been recorded. 


\section{3) Technique:}

\section{A) Ultrasonographic examination:}

It took place while the patient was supine with head and neck in extension position.

Examination was done by ultrasound device (Semens- Acuson p300), which contains both curved $(5 \mathrm{MHz})$ and linear $(7.5 \mathrm{MHz})$ probes. In the preoperative holding area while the patient lying supine with the head and neck in extension position, the sonographic assessment was done. Curved low-frequency (5 MHz) transducer was used to visualize the shadows of the hyoid bone first and then the mandible, then the hyomental distance was measured from the upper border of the hyoid bone to the lower border of the mentum in the head extended position. Then the highfrequency linear probe was placed in the submandibular area in the midline. Without changing the position of the probe, the linear array of the US probe was rotated in the transverse planes from cephalad to caudal, until simultaneous visualization of the epiglottis and posterior part of vocal cords was observed on the screen. obtained:

After examination the following was

1) Visualizing the hyoid bone in the midline sagittal view. On the sagittal and parasagittal views, the hyoid bone is visible in cross section as a narrow hyperechoic curved structure that cast an acoustic shadow.

2) Measurement of the hyomental distance in extended neck position: measured from upper border of hyoid bone to lower border of mentum in head extended position.

3) Measurement of the distance from the epiglottis to the midpoint of the distance between the vocal cords. An oblique transverse US view of the airway can help to obtain this measurement.
All of these data and measurements have been recorded in each patient's sheet. Then the patient was transferred to the operating room. At the operating room, airway devices and anesthesia machine, ventilators, flowmeters and other equipments were checked promptly at the beginning of the day.

After cannulation, monitoring equipments were attached to the patients including 3 lead ECG, noninvasive blood pressure, pulse oximetry and capnogram. Patients were pre-oxygenated with $100 \%$ oxygen for 3 minutes then the induction of anesthesia was achieved by:

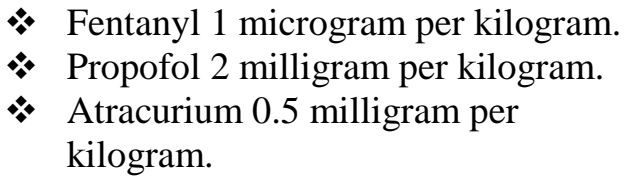

If the patient was expected to be difficult by El-Ganzouri airway risk index ( $>4)$, only fentanyl and propofol were given, then ventilation was assessed. If the ventilation was easy, a muscle relaxant was given.

Atracurium was given to facilitate endotracheal intubation which was done when TOF count was zero by skilled anesthetist who performed all intubation procedures for all patients using Mackintosh laryngoscope blade. The Cormack-Lehane grading was noted and recorded without external laryngeal manipulation.

Any need for external laryngeal compression, use of other assistant tools like (use of an oral airway, gum elastic bougie or stylet). Number of trials of intubation, or any other type of difficulty were also recorded.

\section{4) Ethical Consideration:}

\section{-The study was approved by the Ethics Board of Al-Azhar University.}

- Written signed consent was obtained from all patients before getting them involved in the study.

- There were no harmful effects results from ultrasonography.

- The steps of the study; the aims, the potential benefits 
and dangers all were discussed with patients.

- Patients were informed about any abnormal results of procedures and testes performed.

- Patients had the rights to refuse participation, and withdraw anytime they want.

- Confidentiality of all data and test results of all the study population were preserved.

\section{Statistical Analysis:}

Statistical presentation and analysis of the present study was conducted, using the mean, standard deviation, unpaired student $\mathrm{t}$ test, analysis of variance [ANOVA] test, chisquare, Linear Correlation Coefficient [r] and ROC-curve tests by IBM SPSS Statistics for Windows, Version 20.0 (Armonk, NY: IBM Corp.). Linear correlation coefficient was used for detection of correlation between two quantitative variables in one group. ROCcurve: - Receiver Operating Characteristic curve analysis sensitivity: - Probability that the test results are positive when the disease is present (true positive rate) was expressed as a percentage. Specificity: - Probability that the test results are negative when the disease is present (true negative rate) was expressed as a percentage.

PPV: - Positive Predictive value (probability that the disease is present when the test is positive).

NPV: - Negative Predictive value (probability that the disease is present when the test is negative).

Accuracy: - the ratio of the true positive and true negative on all patients.

$$
\begin{aligned}
& >0.05 \text { Non significant }<0.05 * \text { Significant } \\
& <0.001^{* *} \text { highly significant }
\end{aligned}
$$

\section{Results}

This prospective study was conducted on 60 patients who were randomly selected in different operating rooms in Al-Azhar University Hospitals in the period from July 2018 to September 2019.

Table (1): Comparison between study and control groups as regard age

\begin{tabular}{|c|r|c|c|c|c|c|}
\hline \multirow{2}{*}{ Groups } & \multicolumn{9}{|c|}{ Age (years) } & \multicolumn{2}{c|}{ T-test } \\
\cline { 2 - 5 } & \multicolumn{2}{|c|}{ Range } & Mean \pm SD & t & P-value \\
\hline $\begin{array}{c}\text { Study } \\
\mathrm{n}=30\end{array}$ & 20 & - & 58 & $40.33 \pm 12.47$ & 0.483 & 0.631 \\
\hline $\begin{array}{c}\text { Control } \\
\mathrm{n}=30\end{array}$ & 18 & - & 60 & $38.77 \pm 12.65$ & \\
\hline
\end{tabular}

There was no statistically significant difference between the two groups as regard age (Table 1).

\begin{tabular}{|c|c|c|c|c|c|c|c|}
\hline \multirow{3}{*}{\multicolumn{2}{|c|}{ Sex }} & \multicolumn{6}{|c|}{ Groups } \\
\hline & & \multicolumn{2}{|c|}{$\begin{array}{c}\text { Study } \\
n=30\end{array}$} & \multicolumn{2}{|c|}{$\begin{array}{c}\text { Control } \\
n=30\end{array}$} & \multicolumn{2}{|c|}{$\begin{array}{l}\text { Total } \\
n=60\end{array}$} \\
\hline & & $\mathrm{N}$ & $\%$ & $\mathrm{~N}$ & $\%$ & $\mathrm{~N}$ & $\%$ \\
\hline \multicolumn{2}{|c|}{ Female } & 13 & 43.3 & 14 & 46.7 & 27 & 45.0 \\
\hline \multicolumn{2}{|c|}{ Male } & 17 & 56.7 & 16 & 53.3 & 33 & 55.0 \\
\hline \multicolumn{2}{|c|}{ Total } & 30 & 100.0 & 30 & 100.0 & 60 & 100.0 \\
\hline \multirow{2}{*}{ Chi-square } & $\mathrm{X}^{2}$ & \multicolumn{6}{|c|}{0.067} \\
\hline & $\mathrm{P}$-value & \multicolumn{6}{|c|}{0.795} \\
\hline
\end{tabular}

Table (2): Comparison between study and control groups as regard sex

There was no statistically significant difference between the two groups as regard sex (Table 2). 
Table (3): Comparison between study and control groups as regard easy (EGRI<4) and difficult $(E G R I \geq 4)$ intubation by El-Ganzouri airway risk index in relation to Cormack-Lehane grading

\begin{tabular}{|c|c|c|c|c|c|c|c|c|c|}
\hline \multirow{3}{*}{ Groups } & \multirow{3}{*}{ EGRI } & \multicolumn{8}{|c|}{ C-L Grade } \\
\hline & & \multicolumn{2}{|c|}{ Easy } & \multicolumn{2}{|c|}{ Difficult } & \multicolumn{2}{|c|}{ Total } & \multicolumn{2}{|c|}{ Chi-square } \\
\hline & & $\mathrm{N}$ & $\%$ & $\mathrm{~N}$ & $\%$ & $\mathrm{~N}$ & $\%$ & $\mathrm{X}^{2}$ & P-value \\
\hline \multirow{2}{*}{$\begin{array}{c}\text { Study } \\
\mathbf{n}=\mathbf{3 0}\end{array}$} & Easy & 18 & 60.0 & 4 & 13.3 & 22 & 73.3 & \multirow{2}{*}{16.364} & \multirow{2}{*}{$<0.001 * *$} \\
\hline & Difficult & 0 & 0.0 & 8 & 26.7 & 8 & 26.7 & & \\
\hline \multirow{2}{*}{$\begin{array}{c}\begin{array}{c}\text { Control } \\
\mathbf{n}=\mathbf{3 0}\end{array} \\
\end{array}$} & Easy & 16 & 53.3 & 5 & 16.7 & 21 & 70.0 & \multirow{2}{*}{14.694} & \multirow{2}{*}{$<0.001 * *$} \\
\hline & Difficult & 0 & 0.0 & 9 & 30.0 & 9 & 30.0 & & \\
\hline
\end{tabular}

The table showed statistically highly significant relation (the lower the score the better the visualization) between El-Ganzouri airway risk index as regard easy (EGRI $<4)$ and difficult $($ EGRI $\geq 4)$ intubation in both study and control groups in relation to the corresponding Cormack-Lehane grading (grade I and II was considered easy intubation while grade III and IV was considered as difficult) (table 3).

Table (4): Relation of the ultrasound measured distances and Cormack-Lehane grade in the study group

\begin{tabular}{|c|c|c|c|c|c|c|c|c|}
\hline & \multicolumn{8}{|c|}{ C-L Grade } \\
\hline & \multicolumn{3}{|c|}{ Easy } & \multicolumn{3}{|c|}{ Difficult } & \multicolumn{2}{|c|}{ T-test } \\
\hline & $\mathrm{N}$ & Mean & SD & $\mathrm{N}$ & Mean & SD & $\mathrm{t}$ & P-value \\
\hline $\mathrm{TMD}(\mathrm{cm})$. & 18 & 6.43 & 0.39 & 12 & 6.03 & 0.27 & 3.181 & $0.004 *$ \\
\hline $\mathrm{HMD}(\mathrm{cm})$. & 18 & 6.01 & 0.21 & 12 & 5.71 & 0.28 & 2.296 & $0.029 *$ \\
\hline Epi.toV.C D(cm.) & 18 & 1.87 & 0.13 & 12 & 1.15 & 0.33 & 8.519 & $<0.001 * *$ \\
\hline
\end{tabular}

TMD: thyromental distance, HMD: hyomental distance, Epi.toV.C D: epiglottis to midpoint between the two vocal cords distance.

This table showed statistically highly significant relation (the longer the distance the better the grade) between epiglottis to vocal cord distance and the Cormack-Lehane grade. It also shows statistically significant relation (the longer the distance the better the grade) between thyromental distance and the corresponding Cormack-Lehane grade and also the hyomental distance with the corresponding Cormack-Lehane grade (Table 4).

Table (5): Comparison between different Cormack-Lehane grades and the range of hyomental distance in the study group

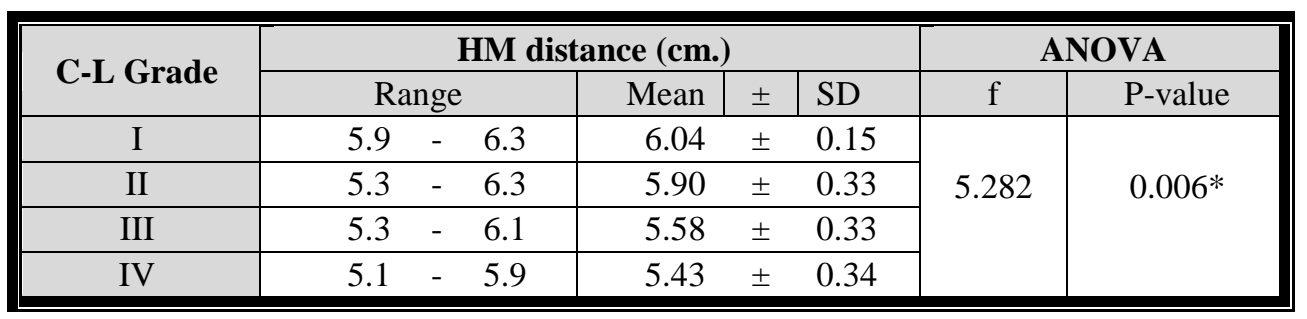

HM distance: hyomental distance.

The table showed statistically significant relation (the longer the distance the better the grade) between range of hyomental distance and corresponding grades of Cormack-Lehane scale (table 5). 
Table (6): Comparison between different Cormack-Lehane grades and the range of epiglottis to vocal cord distance in the study group

\begin{tabular}{|c|c|c|c|c|c|c|c|}
\hline \multirow{2}{*}{ C-L Grade } & \multicolumn{5}{|c|}{ Epi.toV.C distance (cm.) } & \multicolumn{2}{|c|}{ ANOVA } \\
\hline & \multicolumn{2}{|c|}{ Range } & Mean & \pm & SD & $\mathrm{f}$ & P-value \\
\hline I & 1.85 & - 1.98 & 1.92 & \pm & 0.05 & \multirow{4}{*}{4.651} & \multirow{4}{*}{$0.010^{*}$} \\
\hline II & 0.95 & -2 & 1.59 & \pm & 0.45 & & \\
\hline III & 0.98 & -1.76 & 1.36 & \pm & 0.36 & & \\
\hline IV & 0.95 & - $\quad 1.75$ & 1.16 & \pm & 0.39 & & \\
\hline
\end{tabular}

Epi.toV.C distance: epiglottis to midpoint between the two vocal cords distance.

Table (6) showed statistically significant relation (the longer the distance the better the grade) between range of epiglottis to vocal cord distance and corresponding grades of Cormack-Lehane grading scale.

Table (7): Correlation of the study measured distances with the thyromental distance and El-Ganzouri airway risk index in the study group

\begin{tabular}{|c|c|c|c|c|}
\hline \multirow{2}{*}{} & \multicolumn{2}{|c|}{ TMD } & \multicolumn{2}{c|}{ EGRI } \\
\cline { 2 - 5 } & $\mathrm{r}$ & $\mathrm{P}$-value & $\mathrm{r}$ & $\mathrm{P}$-value \\
\hline HM distance & 0.817 & $<0.001^{* *}$ & -0.712 & $<0.001^{* *}$ \\
\hline Epi.toV.C dis. & 0.790 & $<0.001^{* *}$ & -0.694 & $<0.001^{* *}$ \\
\hline
\end{tabular}

TMD: thyromental distance, HM distance: hyomental distance, Epi.toV.C distance: epiglottis to midpoint between the two vocal cords distance.

This table showed statistically highly significant +ve correlation between the study measured distances (HM dist. and Epi.toV.C) with the thyromental distance and statistically highly significant -ve correlation with El-Ganzouri airway risk index (Table 7)

Table (8): ROC analysis, sensitivity and specificity of El-Ganzouri airway risk index between study and control groups

\begin{tabular}{|c|c|c|c|c|c|}
\hline \multicolumn{5}{|c|}{ ROC curve between Study and control as regard EGRI } \\
\hline Cutoff & Sens. & Spec. & PPV & NPV & Accuracy \\
\hline$\leq 3$ & 73.3 & 30.0 & 51.2 & 52.9 & 0.56 \\
\hline
\end{tabular}

ROC curve: Receiver Operating Characteristic curve analysis, Sens.: Sensitivity, Spec.: Specificity, PPV: Positive Predictive value, NPV: Negative Predictive value.

This table showed that the area under curve at ROC analysis was $0.56 \mathrm{CI}$. The sensitivity of El-Ganzouri airway risk index was $73.3 \%$ and the specificity was $30.0 \%$ at cutoff value of 3 above which the incidence of difficulty is high (Table 8).

Table (9): ROC analysis, sensitivity and specificity of Cormack-Lehane grade between study and control groups.

\begin{tabular}{|c|c|c|c|c|c|}
\hline \multicolumn{5}{|c||}{ ROC curve between study and control as regard C-L grade } \\
\hline Cutoff & Sens. & Spec. & PPV & NPV & Accuracy \\
\hline$>2 *$ & 83.3 & 43.3 & 59. & 72.2 & 0.61 \\
\hline
\end{tabular}


ROC curve: Receiver Operating Characteristic curve analysis, Sens.: Sensitivity, Spec.: Specificity, PPV: Positive Predictive value, NPV: Negative Predictive value.

Table (9) showed that the area under curve at ROC analysis was $0.61 \mathrm{CI}$. The sensitivity of CormackLehane grade was $83.3 \%$ and the specificity was $43.3 \%$ at cut off value of 2 above which the intubation difficulty is high.

Table (10): ROC analysis, sensitivity and specificity of Cormack-Lehane grade with hyomental distance in study group

\begin{tabular}{|c|c|c|c|c|c|}
\hline \multicolumn{6}{|c|}{$\begin{array}{l}\text { ROC curve between } \mathrm{C}-\mathrm{L} \text { grade as regards hyomental distance in study } \\
\text { group }\end{array}$} \\
\hline Cutoff & Sens. & Spec. & PPV & NPV & Accuracy \\
\hline$\leq 5.6^{*}$ & 75.0 & 94.4 & 90.0 & 85.0 & 85.0 \\
\hline
\end{tabular}

ROC curve: Receiver Operating Characteristic curve analysis, Sens.: Sensitivity, Spec.: Specificity, PPV: Positive Predictive value, NPV: Negative Predictive value.

This table showed that the area under curve at ROC analysis was $0.85 \mathrm{CI}$. The sensitivity of hyomental distance was $75.0 \%$ and the specificity was $94.4 \%$ at cutoff value of $5.6 \mathrm{~cm}$ below which the intubation difficulty is high (Table 10).

Table (11): ROC analysis, sensitivity and specificity of Cormack-Lehane grade with epiglottis to vocal cord distance in study group.

\begin{tabular}{|c|c|c|c|c|c|}
\hline \multicolumn{5}{||c|}{ ROC curve between C-L grade as regards Epi.toV.C in study group } \\
\hline Cut off & Sens. & Spec. & PPV & NPV & Accuracy \\
\hline$\leq 1.2$ & 75.0 & 100.0 & 100.0 & 85.7 & 0.92 \\
\hline
\end{tabular}

ROC curve: Receiver Operating Characteristic curve analysis, Sens.: Sensitivity, Spec.: Specificity, PPV: Positive Predictive value, NPV: Negative Predictive value, Epi.toV.C: epiglottis to midpoint between the two vocal cords distance.

Table (11) showed that the area under curve at ROC analysis was 0.92 CI. The sensitivity of Epi.toV.C distance was $75.0 \%$ and the specificity was $100 \%$ at cutoff value of $1.2 \mathrm{~cm}$ below which the intubation difficulty is high.

\section{Discussion}

The objective of this study was to compare ElGanzouri airway risk index (EGRI) alone and in combination with upper airway ultrasound in preoperative airway assessment in patients scheduled for elective surgeries.

The present study searched the ability of ultrasound examination of the neck that takes few minutes in combination with El-Ganzouri airway risk index to reach a conclusion as regards the evaluation of the patient's airway and prediction of difficult intubation before induction of anesthesia.

The fast and quick airway assessment can be a good supplementary tool in the busy anesthesiologists' daily work where in the anesthesia care teams can rest assured that before transferring the patients to the operating room they have insightful visuals of the patients' airways that was obtained noninvasively and without much delay in the daily routines of anesthesia care giving.

After reviewing all available publications, till the time of submitting the protocol of that study, three parameters was selected to be combined with El-Ganzouri airway risk index to assess the patient airway before intubation and comparing it with the Cormack-Lehane classification, the parameters of the study are obtained by fast and quick airway assessment by the ultrasound. 
There were no statistically significant differences between the two groups as regard the demographic data. Also as regard easiness of mask ventilation there were no statistically significant differences between the two groups.

Our study showed that according to ElGanzouri airway risk index study group included $22(73.3 \%)$ were easy intubated and 8 (26.7\%) were difficult intubation while the control group included $21(70.0 \%)$ were easy intubated and $9(30.0 \%)$ were difficult. With Pvalue of 0.774 there was no statistically significant difference between the two groups regarding El-Ganzouri airway risk index.

In our study the sensitivity and specificity of El-Ganzouri airway risk index between study and control groups at cutoff value of 3 were $73.3 \%$ and $30.0 \%$ with accuracy $56 \%$ and positive predictive value $51.2 \%$, and this may be explained by the use of direct laryngoscopy not the video laryngscopy. This is the same explanation given by Aziz ${ }^{(10)}$, who stated that despite similar approaches and aims of direct laryngoscopy and video laryngoscopy, both airway management methods are entirely different and probably should have different criteria for difficult airways and therefore should have different methods of difficult airway assessment. Both this statement and results of our study above may lead to possible explanation of moderate predictive value of ElGanzouri risk index in this study. Possible difficulty of direct laryngoscopy not always points out the difficulty of video laryngoscopy, since video laryngoscopy is different from direct laryngoscopy and improves glottis visualization per SE.

According to Cormack-Lehane grading, easy intubation was found in $60 \%$ of patients in the study group compared to $53.3 \%$ in the control group which was statistically non-significant in the present study.

Also our study showed statistically highly significant relation between El-Ganzouri airway risk index concerning easy and difficult intubation in both study and control groups in relation to the corresponding Cormack-Lehane grading with $\mathrm{p}$-value $<0.001$ using macintosh laryngoscopy in the process of intubation. This finding is consistent with a study done by Cortellazzi et al. ${ }^{(8)}$ where data from two subsequent groups of patients, intubated with Macintosh laryngoscopy (ML) and video (VL) laryngoscopy, were retrospectively analyzed and El-Ganzouri airway risk index was taken as index test. The two types of laryngoscopy were adopted as reference for the presence of Cormack and Lehane grading III-IV. The study found that:

$>$ In the ML group, sensitivity and specificity of El- Ganzouri airway risk index were $69.7 \%$ and $66.3 \%$ at the cutoff value of 2 .

$>$ In the VL group, sensitivity and specificity of El- Ganzouri airway risk index were $93.3 \%$ and $76.6 \%$ at the cutoff value of 3 . They also found that the area under curve (AUC) was 0.74 in the ML group, and 0.91 in the VL group; both values were significantly higher than $0.5(\mathrm{P}<0.001)$.

The results of Cortellazzi et al. (8) study suggested that the predictive value of ElGanzouri airway risk index may have been underestimated due to limited accuracy of Macintosh laryngoscopy as reference standard. If accuracy values on the order of those obtained in this study can be reproducibly obtained using glidescope video laryngoscopy as reference, then the El-Ganzouri index may be reconsidered as a decisional tool if video laryngoscopy is used in daily clinical practice. The entire ability of El-Ganzouri airway risk index to predict difficult intubation and difficult ventilation was evaluated using the Receiver Operating Characteristic curve analysis (ROC curve), where the optimal cutoff value of ElGanzouri airway risk index to predict difficult airway was identified using the Youden index method, which defines the cutoff in terms of the maximal sum of sensitivity and specificity.

$>$ At cutoff value 3 , the sensitivity, specificity, positive predictive value, and negative predictive value were of $64.1 \%, 81.4 \%, 36.1 \%$, and $92.4 \%$, respectively and the area under the ROC curve for difficult intubation was 0.77 (95\% CI 0.74-0.80).

$>$ The cutoff value of 4 suggested by ElGanzouri was associated with a sensitivity of $43.6 \%$ and specificity of $91.7 \%$. EGRI registered also a higher ability to predict difficult ventilation (AUC $=0.76$ (95\% CI 0.71- 0.81). An EGRI $\geq 3$ was the optimal cutoff to predict a difficult ventilation with a sensitivity of $66 \%$ and a specificity of $77 \%$. 
The results of our study is consistent with the results of a study done by Antons and Sigita ${ }^{\text {(9) }}$, determining the specificity and sensitivity of El-Ganzouri multivariate risk index to predict difficult laryngeal exposure using C-MAC video laryngoscope. The study was conducted on 29 patients, who were examined by ElGanzouri risk index before induction of anesthesia. After induction of anesthesia video laryngoscopy was performed using C-MAC video laryngoscope and laryngeal visualization was graded using Cormack-Lehane scale, then endotracheal tube insertion was performed and numbers of attempts as well as complications during intubation were recorded. Sensitivity, specificity, positive and negative predictive values were calculated, receiver operating characteristic curve and area under curve was also obtained. Of the 29 patient, 22 were judged to be at high risk of difficult intubation according to El-Ganzouri risk index and of those 5 had C-L grade $\geq 2$ and 17 had C-L grade 1 on laryngeal exposure.

The results of this study shows moderate sensitivity and specificity of ElGanzouri risk index, which means that ElGanzouri risk index can identify the difficult (i.e., $C-L \geq 2$ ) airway patients but at the same time may be falsely-positive in patients with "simple" airways as well. Sensitivity and specificity were $54.2 \%$ and $80.0 \%$ at ElGanzouri risk index cut-off value of 2 points. Calculated positive predictive value was $26.7 \%$ and negative predictive value was $92.9 \%$ and calculated area under curve was $78.3 \%$.

In the research, conducted by Cortellazzi $\boldsymbol{e t}$ al. (8) discussed before, El-Ganzouri risk index sensitivity and specificity when using glidescope video laryngoscope was $93.3 \%$ and $76.6 \%$ respectively at cutoff value of 3 points, which is higher than predictive value of ElGanzouri risk index with the same cutoff, obtained in Anton's study.

As it was concluded in several studies, usage of video laryngoscopes enables much better visualization of glottic opening and increases the possibility of first-attempt intubation in comparison with direct laryngoscopy ${ }^{(\mathbf{1 0})}$.

Our study found that the distances measured by ultrasound were:

$>$ For hyomental distance it was $6.01 \pm$ 0.21 for easy intubation while it was $5.71 \pm 0.28$ for difficult intubation .
$>$ For epiglottis to vocal cord distance it was $1.87 \pm 0.13$ for easy intubation and $1.15 \pm 0.33$ for difficult intubation.

We found that there was a statistically significant relation between hyomental distance and the corresponding Cormack-Lehane grade (p-value of 0.029). Also, there was statistically significant relation between range of hyomental distance $(5.1 \mathrm{~cm}-6.3 \mathrm{~cm})$ and corresponding grades of Cormack-Lehane scale with p-value of 0.006 .

Our study also showed statistically highly significant +ve correlation between the hyomental distance with the thyromental distance with P-value $<0.001$ and statistically highly significant -ve correlation between the hyomental distance with El-Ganzouri airway risk index with P-value < 0.001 . Also, it showed that at Cutoff value $\leq 5.6$ sensitivity and specificity of Cormack-Lehane grade with hyomental distance in the study group was $75.0 \%$ and $94.4 \%$ respectively with accuracy of $85 \%$. In a study done by Nevena et al. ${ }^{(11)}$ in which they examined hyomental distance in the different head positions (at the extreme of head extension (HMDe) and the one in the neutral position (HMDn)) and hyomental distance ratio (HMDR) in predicting difficult intubation in 262 patients that underwent elective surgical operations at the Clinical Centre of Serbia. The patients were divided into two groups with and without difficult intubation based on the Cormack-Lehane grade. They found that no significant statistical difference was observed in the HMDn between the groups with and without difficult intubation, while for the HMDe and HMDR were significantly different between the two groups. The finding of this study is consistent with the finding of our study as regard the sensitivity of the hyomental distance in neck extension position (75.0\% in our study and $75.9 \%$ in Nevena's study).

Aktas et al. ${ }^{(12)}$ in their study reported that out of the 249 patients without difficult intubation, $207(83.1 \%)$ had HMDn $\leq 5.5 \mathrm{~cm}$ and $42(16.9 \%)$ had HMDn $>5.5 \mathrm{~cm}$. In this group of patients, 60 (24.1\%) had HMDe < 5.3 $\mathrm{cm}$ and 189 (75.9\%) had HMDe $5.3 \mathrm{~cm}$ and more. Furthermore, $11(4.4 \%)$ patients had HMDR 1.2 and less and $238(95.6 \%)$ had HMDR more than 1.2. Out of the 13 patients with difficult intubation, 9 (69.2\%) had HMDn $\leq 5.5 \mathrm{~cm}, 8(61.5 \%)$ had HMDe $<5.3 \mathrm{~cm}$, and $9(69.2 \%)$ had HMDR 1.2 and less. The impact 
of all the observed factors on difficult intubation was investigated by applying the univariate logistic regression analysis. The HMDe ( $\mathrm{p}=0.006)$, and HMDR ( $=0.000)$ stood out as statistically significant factors in the univariate logistic regression and were included in the logistic regression model confirming the predictive value of these parameters regarding difficult intubation. This relationship was not observed for the HMDn ( $p$ $=0.209$ ). The HMDR had a sensitivity of $95.6 \%$ and specificity of $69.2 \%$, while the HMDe had sensitivity of $75.9 \%$ and specificity of $61.5 \%$. The sensitivity of the HMDn for predicting difficult intubation was $30.8 \%$ and specificity was $83.1 \%$. Our results are consistent with the findings of a study done by Aktas et al. ${ }^{(12)}$. In our study hyomental distance in head neutral position and in head extension position were measured then hyomental distance ratio was calculated and experienced anesthesiologist blinded to the results of the airway assessments, performed all of the direct laryngoscopies and classified the laryngoscopic view according to Cormack-Lehane grading (CL grading). Easy visualization of larynx (EVL) was defined as grade I and II and difficult visualization of larynx (DVL) was graded as III and IV. Difficult visualization of larynx (DVL) (Cormack-Lehane grade III and IV) was observed in 27 patients (9\%). On correlating HMD at neutral position of head in predicting DVL showed no statistical significance with $p$ value of 0.63 ; whereas extended position HMD of $<6 \mathrm{~cm}$ showed statistical significance with $\mathrm{p}$ value of 0.0018 , which is consistent with the results of our study that showed statistical significance with $\leq 5.6 \mathrm{~cm}$ with $\mathrm{p}$ value of 0.029. Similarly, HMDR (Hyomental Distance ratio) showed significant correlation in prediction of DVL with cutoff of $<1.2$ with $p$ value of 0.0081 .

In another study done by Wojtczak et al. ${ }^{(13)}$ in which five obese and 7 morbidly obese adult patients with a history of either difficult or easy intubation had a submandibular sonographic examination performed in the supine position. The hyomental distance was measured while the head was placed in the neutral position and then in a hyperextended position and the ratio of two distances (hyomental distance ratio) were calculated. The laryngoscopic grades of Cormack-Lehane that were recorded during tracheal intubation were obtained. The mean hyomental distance in the neutral position did not differ significantly between the two groups: $51.3 \pm 5.3 \mathrm{~mm}$ (difficult intubation) versus 57.5 $\pm 4.3 \mathrm{~mm}$ (easy intubation). The difference in the mean hyomental distance in the headextended position was $52.6 \pm 5.8 \mathrm{~mm}$ (difficult intubation) and was $65.5 \pm 4.1 \mathrm{~mm}$ (easy intubation) and differed significantly $(\mathrm{P}<.01)$, which is also consistent with the results of our study in spite of that we excluded obese adult patients from our study. Another univariate random-effects meta-analysis done as a part from identifying risk factors and physical findings that predict difficult intubation, analysis of 3 studies (including 1245 patients) measuring hyomental distance (in neutral position) revealed that with range of $<3-<5.5$ $\mathrm{cm}$ the sensitivity and specificity were $20 \%$ and 97\% respectively ${ }^{(\mathbf{1 4})}$.

For the epiglottis to vocal cord distance, we found statistically highly significant relation between epiglottis to vocal cord distance and the corresponding CormackLehane grade with p-value $<0.001$. Also we found statistically significant relation between range of epiglottis to vocal cord distance and corresponding grades of Cormack-Lehane grading scale with $\mathrm{p}$-value of 0.010 . In the same time, we found statistically highly significant +ve correlation between the epiglottis to vocal cord distance and the hyomental distance and statistically highly significant -ve correlation with El-Ganzouri airway risk index. We also found that the sensitivity and specificity of epiglottis to vocal cord distance at cutoff value of $\leq 1.2 \mathrm{~cm}$ (below which the intubation difficulty is high) with Cormack-Lehane grading scale was $75.0 \%$ and $100 \%$ respectively with accuracy $92 \%$.

In a study done by Deepak $\boldsymbol{e t}$ al. ${ }^{(15)}$ titled ultrasonographic modification of Cormack-Lehane classification for preanesthetic airway assessment which was conducted on patients scheduled for elective surgery and requiring general anesthesia with direct laryngoscopy and endotracheal intubation. In the pre-operative holding area, the following measurements were obtained with the oblique-transverse ultrasound view of the airway: (a) the distance from the epiglottis to the midpoint of the distance between the vocal cords, (b) the depth of the pre-epiglottic space, and (c) the total time taken by the operator to achieve the final ultrasonic image. 
The data were then compared with the Cormack-Lehane classification during direct laryngoscopy in the operating room. Subsequently based on the correlation data, the ultrasonographic modification of CormackLehane classification was developed. It was observed that there was a correlation of the distance between the epiglottis and the vocal cords (E-VC) with the Cormack-Lehane grading. Correlation was strong negative with regression coefficient of -0.966 (95\% CI -1.431 to $-0.501 ; \mathrm{p}=0.0001)$. Subsequently, the correlation of the pre-epiglottis space (Pre-E) with the Cormack- Lehane grading was strong in positive direction with regression coefficient of 0.595 (95\% CI 0.261 to $0.929 ; \mathrm{p}=0.0008$ ). Finally, the ratio of Pre-E and E-VC distances with the Cormack-Lehane grading had the strongest positive correlation with regression coefficient of 0.495 (95\% CI 0.319 to $0.671 ; p$ $<0.0001$ ), which is consistent with the finding of our study as regard epiglottis to vocal cord distance.

\section{Conclusion}

Using of EGRI as a simple bedside predictive score can improve patient safety by detecting the patients with increased likelihood of difficult intubation. Using ultrasonography of airway for prediction of difficult intubation is a very useful, easy and reliable tool of assessment of the airway. Combination of EGRI with epiglottis to vocal cord distance and hyomental distance is a very sensitive and specific tool for prediction of difficult airway and may be superior to standard methods of assessment.

\section{Recommendations}

\section{We recommend the following:}

$>$ Using Combination of EGRI together with ultrasonography of airway (specially epiglottis to vocal cord distance and hyomental distance) for prediction of difficult airway in all elective cases, either anticipated difficult intubation (to confirm the diagnosis) or any other case (to elicit the unanticipated difficult intubation cases).

$>$ Performing the same technique on a larger scale, and sample size, so that, the sample population would give a higher resolution of the newly developed technique of the airway ultrasound.

\section{References}

1. Cook TM, Woodall N, Frerk C et al. (2011): Major complications of airway management in the UK: results of the Fourth National Audit Project of the Royal College of Anaesthetists and the Difficult Airway Society. Part 1: anaesthesia. Br J Anaesth., 106: 617-31.

2. Berkow LC, Ariyo $P$ (2015): Preoperative assessment of the airway. Trends Anaesth Crit Care, 5: 28-35.

3. Adhikari S, Zeger W, Schmier C et al. (2011): Pilot study to determine the utility of point-of-care ultrasound in the assessment of difficult laryngoscopy. Acad Emerg Med., 18: 754-8.

4. Heinrich S, Birkholz T, Irouschek A et al. (2013): Incidences and predictors of difficult laryngoscopy in adult patients undergoing general anesthesia: a singlecenter analysis of 102,305 cases. J. Anesth., 27 (6): 815-21.

5. Nolan JP, Kelly FE (2011): Airway challenges in critical care. Anaesthesia, 66: 81-92.

6. Reddy PB, Punetha P, Chalam KS (2016): Ultrasonography - A viable tool for airway assessment. Indian J Anaesth., 60: 807-13.

7. Kajekar $\mathbf{P}$, Mendonca $\mathbf{C}$, Gaur $\mathbf{V}$ (2010): Role of ultrasound in airway assessment and management. Interv $\mathbf{J}$ Ultrasound Appl Technol Perioper Care, 1: $97-100$

8. Cortellazzi P, Minati L, Falcone $\mathrm{C}$ et al. (2007): Predictive value of the ElGanzouri multivariate risk index for difficult tracheal intubation: a comparison of Glidescopew video laryngoscopy and conventional Macintosh laryngoscopy. Br J Anaesth., 99: 906-11.

9. Antons Z, Sigita K (2017): Prediction of Difficult Tracheal Video laryngoscopic Intubation Using El-Ganzouri Risk Index. Acta Chirurgica Latviensis, 55 (7): 453-458.

10. Aziz MF, Dillman D, Fu R et al. (2012): Comparative effectiveness of the CMAC video laryngoscope versus direct laryngoscopy in the setting of the predicted difficult airway. Anesthesiology, 116: 629-36. 
11. Nevena $K$, Mirko $L$, Biljana $M$ et al. (2016): Hyomental distance in the different head positions and hyomental distance ratio in predicting difficult intubation. Bosn J Basic Med Sci., 16 (3): 232-236.
12. Aktas S, Atalay YO, Tugrul M (2015):

Predictive value of bedside tests for difficult intubations. European Review for Medical and Pharmacological Sciences, 19: 1595-1599.

13. Wojtczak JA (2012): Submandibular sonography: Assessment of hyomental distances and ratio, tongue size, and floor of the mouth musculature using portable sonography. J Ultrasound Med., 31: 523528.

14. Michael ED, Naheed J, Neill KA et al. (2019): Will This Patient Be Difficult to Intubate? The Rational Clinical Examination Systematic Review. JAMA., 321 (5): 493-503.

15. Deepak G, Arvind S, Bryant I et al. (2012): Ultrasonographic modification of Cormack-lehane classification for peranesthic airway assessment. MEJ Anesth., 21 (6): 36-45. 